\title{
Preparing for Renal Replacement Therapy in Patients with the Ebola Virus Disease
}

\author{
Sarah Faubel ${ }^{a}$ Harold Franch $^{b}$ Anitha Vijayan ${ }^{c}$ Michelle A. Barron ${ }^{d}$

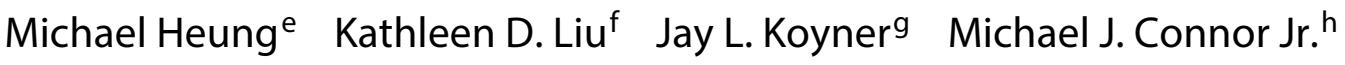 \\ a University of Colorado Denver and VA Medical Center, Renal Division, Denver, Colo., ${ }^{b}$ Emory University School \\ of Medicine, Division of Renal Medicine, Research Service, Atlanta Department of Veterans Affairs Medical Center, \\ Atlanta, Ga., ' Washington University St. Louis, St. Louis, Mo., dUniversity of Colorado, Denver, Division of Infectious \\ Diseases, Denver, Colo., 'University of Michigan, Department of Medicine, Division of Nephrology, Ann Arbor, Mich., \\ ${ }^{\mathrm{f}}$ Departments of Medicine and Anesthesia, University of California, San Francisco, San Francisco, Calif., ${ }^{9}$ Section of \\ Nephrology, Department of Medicine, University of Chicago, Chicago, Ill., ${ }^{\text {h}}$ Divisions of Pulmonary, Allergy and Critical \\ Care \& Renal Medicine, Emory University School of Medicine, Atlanta, Ga., USA
}

\section{Key Words}

Renal replacement therapy - Ebola virus disease .

Biocontainment facility

\begin{abstract}
The Ebola virus disease (EVD) is a serious illness characterized by fever, severe vomiting and diarrhea, and, in severe cases, multi-organ failure requiring mechanical ventilation and renal replacement therapy. The current outbreak has centered in West Africa and affected over 15,000 individuals. EVD is transmitted by direct contact with blood or other infectious bodily fluid, and as such, numerous heath care workers caring for patients with EVD have become infected. During the current outbreak, a number of patients have received advanced supportive care for EVD in Europe and North America and therefore survived. Now, many hospitals in Europe and North America are planning to accept care for patients with EVD. In this review, we discussed the key issues related to the planning and delivery of advanced supportive care in patients with EVD with a focus on the factors necessary to provide renal replacement therapy (RRT). Since success in the treatment of patients with EVD rests on both pa-
\end{abstract}

tient outcome and prevention of transmission of disease to health care workers, we extensively discussed the modes of Ebola virus transmission and recommended protocols to protect health care workers. Experience now indicates that with appropriate planning and protocols, it is possible to successfully treat EVD patients with advanced supportive care (mechanical ventilation and RRT) while avoiding transmission to health care providers.

Video Journal Club "Cappuccino with Claudio Ronco" at http://www.karger.com/?doi=371530. @ 2015 S. Karger AG, Basel

\section{Introduction}

Ebola virus disease (EVD) was first identified in 1976. Between 1976 and 2012, multiple, small-scale EVD outbreaks with a total of approximately 1,700 confirmed cases occurred - primarily in isolated remote villages of Central Africa [1-3]. The current EVD outbreak is centered in West Africa primarily affecting Guinea, Liberia, and Sierra Leone and is thought to have begun in Guinea in December 2013. As of January 7, 2015, the World

\section{KARGER 125}

(2015 S. Karger AG, Basel

$0253-5068 / 15 / 0384-0276 \$ 39.50 / 0$

E-Mail karger@karger.com

www.karger.com/bpu
Sarah Faubel, MD

University of Colorado Denver and Denver VA Medical Center

International Medicine, Renal Division

12700 East 19th Ave, Box Cs81, Aurora, CO 80045 (USA)

E-Mail sarah.faubel@ucdenver.edu 
Health Organization (WHO) reports that there have been 20,747 confirmed, probably, \& suspected cases and 8,235 confirmed reported deaths in nine countries (Liberia, Sierra Leone, Guinea, Nigeria, Mali, Senegal, Spain, United Kingdom, and the United States of America) [4]. The current 2014 EVD outbreak is the largest and most complex outbreak of EVD in history and the first to involve large cities and national capitals $[1,4]$. In this outbreak, EVD has been treated in resource-rich settings of Europe and North America for the first time. As of January 5, 2015, there have been at least 24 cases treated in Europe and North America with the majority of these cases having been evacuated health care workers diagnosed with the infection in West Africa. However, there have been two documented imported cases diagnosed in the United States and there have been three known cases of secondary infections of health care workers ( 1 in Spain and 2 in the United States).

\section{EVD Natural History and Critical Illness Preparations}

EVD is accompanied by a severe hemorrhagic fever with a historically high fatality rate approaching $70-90 \%$ in previous outbreaks and, during the current outbreak, case fatality rates in West Africa have ranged from 40$70 \%$ based on the availability of resources for supportive care [5]. In resource-rich settings, 5 of the 24 patients have died (21\% mortality).

Unfortunately, despite the extent of the current outbreak, there is limited data describing the natural history of severe EVD when treated in resource-rich settings. From our experience to date, it is clear that there are a subset of patients with EVD who are likely to develop a severe form of this disease, characterized by fulminant hypoxic respiratory failure, acute kidney injury (AKI), encephalopathy, and occasionally severe shock and severe metabolic acidosis (T. Uyeki, G. Weinstein, A. Kalil personal communications).

\section{Importance of Nephrology Involvement}

Many features of EVD indicate that the active involvement of nephrologists in patient care will be needed due to the possibility of: (1) multiple electrolyte abnormalities, (2) massive volume losses, and (3) acute kidney injury that may require renal replacement therapy. A hallmark of the current Zaire EVD has been severe voluminous diarrhea, often associated with anorexia, nausea and
Table 1. Differential diagnosis of AKI in Ebola

\author{
Pre-renal azotemia \\ Volume depletion \\ Increased vascular permeability \\ ATN \\ Adrenal insufficiency \\ Shock \\ Cytokine storm \\ Superinfection with bacterial pathogens or malaria \\ Experimental therapeutic agents \\ Rhabdomyolysis \\ Antibiotics \\ Direct infection of tubular cells \\ Vascular \\ Disseminated intravascular coagulation \\ Retroperitoneal hemorrhage \\ Abdominal compartment syndrome
}

vomiting [6]. A systemic inflammatory response may ensue in severe cases resulting in increased vascular permeability, reduced serum albumin, and third spacing of fluid [7]. Patients may require massive volume resuscitation, and weight gains between 10 and $15 \mathrm{~kg}$ are not unusual with adequate fluid management. Hyponatremia, hypocalcemia, hypokalemia, hypomagnesemia, and both lactic and non-gap metabolic acidosis have been reported as common. Adequate fluid and electrolyte management contribute to reduced mortality in patients treated in developed countries and has the potential to improve current outcomes in West Africa [6].

In African Ebola outbreaks, AKI appears to be common and associated with increased mortality, but the lack of laboratory testing in most treatment centers makes the true incidence unknown. The best data come from the 2000 Sudan Ebola virus outbreak in Uganda where fatal cases had a higher mean serum creatinine and BUN than survivors [8]. In the current outbreak, the physiology of the disease and case reports of AKI responding to volume resuscitation have led to the idea that pre-renal azotemia from massive volume losses is common in West Africa.

In addition to hypovolemia, AKI secondary to acute tubular necrosis has been reported in patients who are adequately volume resuscitated, including patients who have not had hypotension [9] (B. Wall personal communication). These patients have been severely ill and have had high levels of viral RNA in their blood. We can only speculate as to the cause of AKI in these patients (table 1). Disseminated intravascular coagulation occurs during EVD with potential for kidney injury. Direct viral infec- 
tion of renal tubular cells has been reported in pathology case series of Marburg- and Ebola-infected patients and may contribute to AKI [10]. Viral illnesses are associated with rhabdomyolysis and an AST greater than ALT predominance has been noted on laboratory testing in EVD patients [8]. Obviously, any cause of AKI that could occur in any critically ill patients may also occur in EVD. In particular, bacterial superinfection has been reported [11]. AKI may be severe enough to require renal replacement therapy, as was performed in 3 patients in the United States and at least one patient in Germany. With the recent report that a patient recovered after prolonged dialysis for AKI with EVD [9], it is clear that RRT may be a useful treatment for supportive care in severe cases of this disease and cannot definitively be considered futile care.

There remains no approved EVD-specific treatment regimen beyond advanced supportive care. However, there is general agreement that with aggressive supportive care, the mortality rate in resource-rich settings will be much lower than in resource-poor settings. Advanced supportive care will initially consist of aggressive fluid resuscitation and close electrolyte monitoring and replacement. Should organ failure ensue, mechanical ventilation and RRT may be required. Given the possibility of multiorgan failure, hospitals in resource-rich settings should consider planning that includes the ability to provide critical care services, including RRT (preferably continuous renal replacement therapy). Indeed, the U.S. Centers for Disease Control (CDC) has made the general recommended that 'Inpatient care of patients with Ebola should be provided in a hospital with capacity to perform continuous renal replacement therapy (CRRT)' (http://www. cdc.gov/vhf/ebola/hcp/guidance-dialysis.html).

When providing and planning for delivery of RRT and other critical care services in EVD, maintaining the safety of health care workers is of paramount importance and delivery of critical care services must be designed to promote health care worker safety. Since the successful treatment of patients with Ebola virus disease rests on both excellent care of the patient and prevention of transmission to health care workers, the most important factor for preparation in terms of protecting health care workers is a clear understanding of how Ebola virus is transmitted as this guides virtually all aspects of hospital care (e.g., the personal protective equipment needed, the nature of the biocontainment room, the necessity of dedicated equipment for the patient). With adequate training, preparation, and adherence to safety protocols, experience suggests that it is possible to provide advanced life support safely [9].

\section{How Is Ebola Virus Transmitted?}

Ebola virus is transmitted to humans via contact with the body, blood or bodily fluids from infected patients. Blood, vomit, stool, urine, saliva, sweat, and semen are all potentially infectious bodily fluids, although the infectivity of these fluids varies during the course of the disease [12]. Specifically, infectious bodily fluids are most likely to transmit the disease late in the course of the Ebola virus disease when viral loads are the highest. The higher the viral load, the higher the risk of transmission. Like other blood-borne illnesses, direct blood to blood contact can transmit the disease, and transmission via needle stick injury has been reported [13]. Additionally, mucous membranes or skin breaks can be other modes of entry. There is a case report of transmission through sexual contact [14].

Unlike influenza A, measles, or tuberculosis, Ebola virus is not a respiratory-borne illness and airborne infection does not occur. With influenza $\mathrm{A}$, and other airborne diseases, the lung is the primary site of infection and during exhalation infectious droplet nuclei can be released and carried long distances in the air (greater than 10 meters). These droplet nuclei (particles less than $5 \mu \mathrm{M}$ ) may then be inhaled by other people, thereby causing infection to develop in them. Ebola virus does not transmit disease in this manner. However, aerosolized droplets of any infectious bodily fluid can be created and these particles are infectious. Aerosolization is a non-specific term that refers to a process where liquid particles become airborne. Aerosolized droplets may be created during routine and critical care of hospitalized patients with EVD such as during suctioning, flushing infectious waste down the toilet, etc. These aerosolized droplets are large $(>10 \mu \mathrm{M})$ and can travel only short distances (about 1 meter); however, if these droplets come into contact with the mucus membranes (eyes or mouth, for example) transmission can occur.

Additionally, the Ebola virus can live for 2 to 6 days on surfaces and contact with surfaces exposed to bodily fluids can also transmit the virus. In particular, infection can occur via contact with contaminated clothes or linens of an infected person.

\section{When Is Ebola Virus Transmitted?}

Overwhelming data and experience indicate that persons with EVD are infectious only when symptomatic. Fever is one of first symptoms heralding infection and infectivity. Other initial symptoms include fatigue, malaise, anorexia, myalgia, and abdominal pain; symptoms may occur between 2 and 21 days after exposure, with a 
mean delay of about 8 to 10 days (http://www.cdc.gov/ vhf/ebola/symptoms/). It should be noted that blood tests for Ebola virus (viral antigen and nucleic acid) are generally not positive until 3 days after the onset of symptoms [14]. Thus, a negative test early after symptoms does not rule out the presence of Ebola virus infection. Likewise, a negative test prior to the onset of symptoms is meaningless, and does not rule out Ebola virus infection.

\section{How Infectious Is the Ebola Virus?}

The likelihood of a transmission of the Ebola virus from one person to another is dependent on two factors (1) exposure to infectious bodily fluids and (2) the viral load contained in the bodily fluids. As the disease progresses from fever and malaise, to severe vomiting and diarrhea, to critical illness with organ failure, viral loads rise and may be as high as $1 \times 10^{9}$ copies per $\mathrm{ml}$ of blood [15]. Thus, it is the very sickest person who is most likely to transmit the Ebola virus to contacts. With these factors in mind, it is clear that the risk of transmission to the general public is very low as exposure to infectious bodily fluids is unlikely; in contrast, the risk to health care workers (without proper personal protective equipment) is substantial, as exposure to infectious bodily fluids during the routine care of patients is common (e.g., blood draws, collecting and disposing of bodily fluid waste, suctioning, etc.). Transmission risk to the general population versus health care workers is exemplified by the case of Thomas Duncan (in Dallas, Texas) who had contact with dozens of people prior to hospitalization, and none of these contacts were infected. While symptomatic with fever vomiting and diarrhea, he lived with family members and none became infected. While critically ill, well over 100 health care/hospital workers came in contact, and two nurses were infected. Thus, close contact of health care workers without adequate PPE during severe illness is the most likely scenario for transmission.

Of interest, in the outbreak in Uganda from August 2000-January 2001, RT-PCR was used to compare viral loads in patients with fatal and nonfatal outcomes. RNA copies were $2 \log _{10}$ higher in patients who died compared to those who survived. In addition, viral loads were the highest (over $8 \log _{10}$ RNA copies/ml serum) on days 3-5 post-onset of symptoms [15]. These very high viral loads increase risk for transmission after exposure to blood or bodily fluids, and are higher than for other acute viral infections such as HIV (at $1 \times 10^{6}$ per $\mathrm{ml}$ of blood) [16].

Preparing for RRT in Ebola Virus Disease
What Is the Appropriate Personal Protective

Equipment (PPE) for Health Care Workers Caring for

Patients with Ebola Virus Disease?

Current recommendations for PPE are the following: (1) no skin exposure, (2) respiratory precautions, (3) training in the donning and doffing of PPE, (4) supervised donning and doffing of PPE, (5) hand washing between different PPE doffing steps, and (6) showering after PPE removal if in room for extended period of time or after a high-risk procedure. Detailed recommendations on the types of PPE and the specific order of PPE donning and doffing may be found at the CDC website: http:// www.cdc.gov/vhf/ebola/hcp/procedures-for-ppe.html. It is important to keep in mind that PPE procedures should be standardized and that training and ongoing drilling of proper PPE donning and doffing is essential. Direct supervision and verbal instructions through each step of PPE donning and doffing from a written checklist by a 'buddy' is highly recommended and decreases risk of errors related to fatigue, stress, etc.

As discussed, Ebola virus is not spread via airborne routes; however, respiratory precautions are recommended for health care workers in case an aerosol-generating procedure occurs during the routine care of hospitalized patients (e.g., suctioning). Recommended PPE for respiratory protection includes either a powered air-purifying respirator (PAPR) or an N95 or higher respirator. Skin protection may be achieved with the use of fluidresistant or impermeable gowns, mid-calf boot covers, double gloving, etc; the key is that no skin (including face) should be exposed.

\section{The Need for Care in a Biocontainment Unit}

Due to the high risk of transmission to health care workers and other hospital personnel and patients, the care of patients with EVD disease should occur in an isolated room. Isolation for Ebola patients may occur in either dedicated or improvised biocontainment units (http://www.cdc.gov/vhf/ebola/hcp/infection-preven tion-and-control-recommendations.html). While dedicated biocontainment units [17] designed for any infectious disease are useful, many single-patient rooms containing a private bathroom may be able to be converted for this use. Rooms with windows that facilitate patient monitoring by staff are preferred; however, video monitoring may also be used. For RRT, an intensive care unit (ICU) room is preferred due to the greater number of electrical connections. If the patient is critically ill and may need to be mechanically ventilated, an airborne infection isolation room is strongly preferred to 
deal with aerosols created by intubation and suction. Outside the room, areas for donning and doffing PPE, staff showers, a separate room for point-of-care laboratory testing and waste holding should be established. A place for personnel to be stationed to observe the appropriate and consistent use of PPE and other infection control practices is helpful. Finally, the unit must be separated from the remainder of the hospital so that no one has to enter the unit unnecessarily.

A biocontainment unit relies on the development and constant refinement of specific operating procedures (SOPs) for staff safety. SOPs will vary from hospital to hospital, but starting suggestions may be found at the Emory University website (http://www.emoryhealthcare. org/ebola-protocol/ehc-message.html). Reducing exposure to bodily fluids underlies the SOPs with goals including the following: reducing the number of people who enter the room, reducing the movement of equipment in and out of the room, and reducing the amount and number of times that workers are exposed to blood or bodily fluids. For example, most units have one nurse performing all aspects of care from physical therapy to critical care services such as RRT. To enable the nurse to perform all these functions, extensive training is required on PPE use, infection control procedures, operation of all equipment in the isolation room including CRRT and continuous quality improvement processes.

\section{Laboratory Testing and Medical Equipment in the Biocontainment Unit}

While patients are in biocontainment rooms, access to blood testing and advanced imaging techniques will be limited. Point-of-care testing has generally been accepted as the most effective and readily available option for laboratory testing, but the panel of available studies is limited [18]. Because of the limited number of laboratory tests available with point-of-care testing, some hospital may wish to develop protocols for sending blood and other samples to the general laboratory; in particular, centers should address if and how samples will be tested for culture. Furthermore, if possible, for diagnostic and therapeutic reasons, we recommend dedicated access to portable bedside ultrasound machines with a variety of probes for bedside diagnostic studies and procedural support of vascular access. Additionally, portable plain film radiograph (X-ray) support with dedicated machines can prove useful.

Any medical equipment that is used in the care of an EVD patient should remain sequestered in the patient's biocontainment isolation room until the patient is dis- charged. Then, the equipment and the room should undergo a terminal cleaning and disinfection protocol as established by one's local hospital and regulatory health agencies; general guidelines [19] and specifically directed guidelines for the disinfection of dialysis machines [20] are available.

The Ebola virus does not appear to survive on fomites for more than 2-6 days and is generally susceptible to standard disinfectants with activity against enveloped or non-enveloped viruses (i.e., norovirus, rotavirus, adenovirus, poliovirus) $[12,19]$. It should be noted that it is currently not recommended to dispose or discard durable medical equipment such as mechanical ventilators and RRT machines after use in EVD. Rather, after appropriate disinfection and a recommended rest period of 7-10 days, it is accepted that equipment (e.g., ultrasound machines, mechanical ventilators, hemodialysis machines, portable $\mathrm{X}$-ray machines) can return to general circulation and be used in the care of other patients.

\section{Health Care Worker Planning}

There are many aspects of the care of patients with EVD that affect health care workers. First, it needs to be established as to who will care for patients with EVD. Most health care centers have asked for volunteers among their providers and staff. This includes but is not limited to physicians, nurses, respiratory therapists, laboratorians, and environmental service workers. It is crucial to identify those that are willing to participate in the care of EVD patients early on in the preparedness process to ensure that they receive adequate training in PPE and have the opportunity to become familiar with the designated isolation unit. However, it is important to recognize that not all volunteers may have the skills, training, or attention to details needed to care for patients in the biocontainment facility and that one person not following protocols places many other people at risk. Therefore, the team of providers must be carefully selected from the volunteers. For example, many centers do not use environmental services to routinely clean the biocontainment isolation room. Rather, the clinical staff perform this duty so as to not increase the exposure of additional hospital staff to the isolation environment and transmission risk.

Identifying essential bedside personnel is important in stratifying training. Certain clinical consultants (e.g., infectious diseases, nephrology) may provide important input into the ongoing patient management but would not likely be required to be physically present at the patient's bedside and should be discouraged from physically examining the patient or entering the patient's room unless ab- 
solutely necessary. Use of technology to aid with clinical care and communications should be considered in order to limit the number of individuals with potential exposure.

In addition, discussions with hospital administration, human resources and employee health are important to determine how workload will be distributed when the volunteers are pulled from their regular clinical duties and how compensation and leave will be managed during this time. Issues of return to work, what happens if there is an exposure, and other psychosocial needs of the providers should be discussed and guidance from the CDC, WHO, and regional health departments should be utilized. The CDC has stated the following regarding the activities of health care workers: 'Healthcare workers who provide care to Ebola patients in U.S. facilities while wearing appropriate PPE and with no known breaches in infection control are considered to have low (but not zero) risk of exposure because of the possibility of unrecognized breaches in infection control and should have direct active monitoring. As long as these healthcare workers have direct active monitoring and are asymptomatic, there is no reason for them not to continue to work in hospitals and other patient care settings. There is also no reason for them to have restrictions on travel or other activities. Review and approval of work, travel, use of public conveyances, and attendance at congregate events are not indicated or recommended for such healthcare workers, except to ensure that direct active monitoring continues uninterrupted'. (Interim U.S. Guidance for Monitoring and Movement of Persons with Potential Ebola Virus Exposure, 11/28/2014, http://www.cdc.gov/vhf/ ebola/exposure/monitoring-and-movement-of-personswith-exposure.html).

Finally, the ethical framework for the best practices of care for the potential or known infectious patients should also be considered. For example, some centers have decided against offering cardiopulmonary resuscitation in settings where survival of the patient is likely to be low, yet the risk to health care workers is likely to be high (i.e., high risk for PPE donning errors due to the urgency needed to enter the patient room and high risk of bodily fluid exposure).

\section{Guidelines for Preparing for RRT in Patients with \\ Ebola Virus Disease}

Given the potential need for RRT in patients EVD and the added complexity of performing RRT in a biocontainment unit, early and active involvement of nephrologists in hospital planning is essential. The most important guiding principle for planning RRT in EVD is that center- specific expertise, experience, and creativity should guide protocol development. Protocols will incorporate local experience regarding aspects of RRT (e.g., the type of anticoagulation) as well as center-specific issues relating to EVD care in general (e.g., type of point of care testing available). Thus, the 'right protocol' at one center may not be the same as the 'right protocol' at another center. It is very important that the number of new procedures and protocols be kept to a minimum, as this can increase risk for medical errors with potential patient harm and risk to the health care worker.

There are 5 specific considerations that affect the RRT protocol in EVD that centers should consider: (1) modality, (2) line placement, (3) lab tests, (4) anticoagulation, and (5) effluent disposal.

\section{Modality of RRT in EVD}

Most EVD patients developing AKI requiring RRT will be critically ill, and therefore, continuous renal replacement therapy (CRRT) is favored over intermittent hemodialysis (IHD) to provide for improved hemodynamic stability and optimal fluid and solute management. Peritoneal dialysis (PD) may be considered relatively contraindicated due to frequency of gastrointestinal involvement in EVD, with substantial risk for bacterial translocation leading to peritonitis. However, acute PD may be a consideration in the pediatric population where vascular access options for hemodialysis may be limited. In addition, PD may be an important option for patients in resource-limited regions of the world where alternative modalities are not readily available [21]. However, it should be noted that, in contrary to RRT effluent, PD effluent will be highly infectious which will further limit the use of acute PD in adults in resource-rich settings.

Beyond patient care aspects, CRRT provides advantages over IHD in regard to health care worker safety. First, in most medical centers, CRRT is provided by trained ICU nurses, whereas IHD is typically performed by dedicated dialysis nurses. Since ICU nurses will usually already be involved in the care of EVD patients, the use of CRRT can reduce the overall exposure of the health care worker by eliminating the need for hands-on involvement by nurses performing dialysis [9]. Second, compared to IHD, CRRT requires fewer circuit changes and generates less waste (both in the form of disposables, such as filter and tubing, and effluent). Again, this reduces the possibility of exposure of health care workers to infectious waste materials.

As patients with AKI requiring dialysis begin to recover and become more hemodynamically stable, the 
usual transition will be to an intermittent modality. In the case of the EVD patient, this can be accomplished by continuing to use the same CRRT machine at an augmented prescription to provide sustained low-efficiency dialysis (SLED) or prolonged intermittent renal replacement therapy (PIRRT). This avoids the need for contamination of a second dialysis machine and obviates the need for additional health care worker (i.e., dialysis nurse) involvement. In addition, IHD machines utilize internal pathways (e.g., for dialysate generation) and non-disposable components, which are at risk for becoming contaminated [20]. Conversely, CRRT machines utilize closed and fully disposable filter circuits. Therefore, use of the same CRRT machine is advisable until the patient recovers adequate renal function to discontinue renal replacement therapy or until the patient is no longer infectious (i.e., blood free of Ebola virus).

\section{Line Placement}

Key considerations regarding line placement include: (1) site, (2) who should place, and (3) method of placement. Line placement for RRT is one of the highest risk procedures for health care workers since exposure to large quantities of blood during routine placement may occur, and accidental blood exposure from aerosolization, splashing, or a needle stick is also possible. Thus, successful placement of a long-working catheter is important for the safety of health care workers.

\section{Site}

We suggest that KDIGO guidelines regarding access site placement for RRT in AKI in general, apply to the care of patients with EVD [22]. Namely, the right internal jugular (R IJ) is the first preferred site, followed by the femoral sites, with the last preferred option being the left internal jugular (L IJ); the subclavian site should be avoided. This order of preference is based on the functionality of the catheter, with the R IJ having the best performance characteristics. Stool output volume may also factor in site selection. If the patient is still experiencing voluminous diarrhea, femoral sites may be less desired unless the bowel management system is in place.

Selection of the (R or L) IJ site requires that X-ray testing is available to confirm appropriate line placement. Some centers may have dedicated X-ray equipment in the biocontainment room to be used exclusively for the EVD patient for the duration of the hospital stay, thus R or L IJ may be reasonable in this setting. If $\mathrm{X}$-ray confirmation of line placement is not available, we suggest the placement of femoral catheters. We are strongly against the use of L or R IJ catheters for RRT if X-ray confirmation is not available; if extraordinary circumstances dictate this approach, then additional informed consent clearly addressing risk and benefit would need to be obtained. Of note, with skilled ultrasonographers, it may be possible to confirm the depth of the IJ catheter with bedside ultrasound of the IVC and might be an option to confirm placement without bedside X-rays. An additional consideration regarding line placement in the R or L IJ site, is that complications of line placement need to be anticipated - specifically, materials for chest tube placement need to be in the room, in case a pneumothorax should occur.

\section{Operator and Method of Placement}

We recommend the placement of temporary dialysis catheters by health care workers with abundant experience, within the isolation room, and with the use of ultrasound guidance in order to minimize complications. Ultrasound guidance is especially important in order to minimize bleeding complications which will be difficult to assess or manage, that is, it is unlikely that patients with EVD will be able to leave the isolation unit safely in order to have radiologic tests (e.g., to evaluate bleeding with a CT or MRI) or undergo IR or surgical procedures (e.g., to repair a bleeding vessel). Once used, the ultrasound - like all similar equipment - should remain in the room until the patient is discharged and at that time the machine can be terminally disinfected, rested, and then re-enter general hospital use.

\section{Lab Tests}

The general approach to laboratory testing in patients with known or suspected EVD is to perform point-ofcare testing. With point-of-care testing, personnel who are already involved in direct patient care can carry out testing, and potential exposure during transport and to laboratory staff can be eliminated. However, the use of point-of-care testing reduces the types of tests available. In general, point-of care testing is available for a basic metabolic panel, ionized calcium, blood gas, liver function tests, and PT/INR. It is likely that point-of-care testing for magnesium, phosphorus, and PTT will not be available. Thus, some hospitals have established protocols where a courier will transport the samples in a biohazard container directly to the lab. Clinicians must consider carefully what labs are essential to provide CRRT under these circumstances. For example, if phosphorus monitoring is not possible, it may be reasonable to empirically supplement phosphorus based on CRRT as hy- 
pophosphatemia may be common and is more life threatening than hyperphosphatemia. Along the same lines, anti-coagulation protocols may need to be modified based on laboratory testing available. In addition to the types of labs available, clinicians should consider how often laboratory studies are needed and consider decreasing the frequency of 'standard' laboratory studies.

\section{Anticoagulation}

The most important consideration in EVD regarding anticoagulation use for CRRT is that filter life needs to be maximized. The importance of maximizing filter life by reducing clotting events cannot be overstated as this can reduce the exposure of health care workers to blood and minimize the need for blood transfusion for the patient. We agree with the KDIGO clinical practice guidelines for RRT in AKI in general (5.3.2.2 Grade 2B) [22] that regional citrate anticoagulation (RCA) is the anticoagulation method of choice when performing CRRT in EVD. This is not to say that centers without RCA cannot reasonably provide CRRT in EVD. Indeed, some centers with RCA protocols may abandon RCA use in EVD if their protocols rely on intense monitoring of ionized calcium (e.g., an excess number of blood draws may unfavorably expose health care workers to excess blood exposure). That said, RCA has many advantages in the care of EVD. For example, for patients with active bleeding, it may be necessary to limit or avoid systemic anticoagulation; thus, RCA may provide an important balance in terms of minimizing patient bleeding risk and maximizing filter life. A recently published case report demonstrated that RCA was used safely and that it provided excellent filter life (60-72 $\mathrm{h}$ per filter) while performing CRRT in a patient with EVD [9]. Since RCA works as an anticoagulant through the binding of calcium and the subsequent inhibition of the coagulation cascade, monitoring of serum ionized calcium is necessary. In an ideal scenario, pre-existing RCA protocols should be adapted to minimize the need for lab monitoring while not exposing the patient to any increased risk due to errors from protocol deviation.

If bleeding is not present, unfractionated heparin and low molecular weight heparin protocols may be used to extend filter life $[23,24]$. Regional heparin with or without post filter protamine, has also been shown to increase filter life [25]. Published protocols for pre-filter heparin have ranged from 1,000 to 1,500 units/h of heparin with protamine being infused at $10-12 \mathrm{mg} / \mathrm{h}$ [24]. The need for frequent blood draws to measure activated partial thromboplastin time (aPTT) both post-filter and systemically may limit their applicability in those with EVD.
However, as with RCA protocols, the modifications to established lab draw schedules will need to be considered.

Beyond anticoagulation, there are a variety of maneuvers that can be attempted with the goal of prolonging filter life. Increasing the blood flow rate above $300 \mathrm{ml} / \mathrm{min}$ will ensure adequate blood delivery to the filter while promoting solute clearance and minimizing the risk of clotting. Additionally for those performing CRRT with convective modalities (either Continuous Veno-Venous Hemo-filtration $(\mathrm{CVVH})$ or Continuous Veno-Venous Hemodiafiltration (CVVHDF), pre-filter replacement fluid has been demonstrated to extend filter life and should be strongly considered. Pre-filter dilution-convective modalities provide lower solute clearance rates compared to post-filter or diffusive modalities by about $15 \%$ [26]; to compensate for this decreased solute removal, increases in replacement fluid rates can be considered. Limited data point to improved filter life with pre-dilution CVVHDF over CVVH [27]; but these data are not definitive and modality selection should be driven by institutional expertise and comfort. As with all patients receiving CRRT, dose of CRRT should be assessed to target effluent volume of $20-25 \mathrm{ml} / \mathrm{kg} / \mathrm{h}$ [22].

\section{Effluent Handling and Disposal}

It is highly unlikely that the dialysis effluent will contain Ebola virus. High flux dialyzers used in most CRRT machines are generally not permeable to substances above $60-70 \mathrm{kD}$ [28]. Ebola virus is an elongated Filoviridae that is about 80 nanometers in diameter, $4,200 \mathrm{kD}$ in mass and up to $14,000 \mathrm{~nm}$ in length. Thus, the large size of the virus should prevent it from moving across the filter into the effluent. In one study, Ebola virus was undetectable by PCR on 3 separate samplings of CRRT effluent, while concurrent plasma and urine samples still showed low-to-medium viral loads [9]. However, effluent is handled in an Ebola positive environment (which may allow for secondary contamination of effluent) and, rarely, dialyzer leaks may allow the viral particles to cross into the effluent. Therefore, RRT effluent should be handled cautiously to mitigate risk of transmission.

CRRT machines utilize one of two options for disposal of the effluent. One method involves the collection of effluent in bags which are then removed and replaced by an empty bag by the bedside nurse as they become full. Typically, the 5L effluent bags are removed by the nurse, manually compressed, forcing effluent to drain into the sink or toilet and bags are reused. The alternative method is to have a disposal line that carries the effluent from the 
CRRT machine to the sink or the toilet. The effluent line runs directly from the machine and is secured to the side of the toilet or drain.

In a patient with EVD, existing center-specific protocols for CRRT can be strengthened to minimize transmission risk. The CDC recommends that untreated effluent be disposed of in the toilet in a manner that prevents splashes (http://www.cdc.gov/vhf/ebola/hcp/guidancedialysis.html). If the center utilizes machines with effluent bags, these bags can be hung near the toilet on a hook or IV pole and the drainage tubing can be connected to the bag, allowing it to empty into the toilet. This negates the need for manual compression of the bags by the nurse. The empty bags should be discarded with the biohazard waste and incinerated, preventing reuse. Centers that utilize machines with drainage lines can continue to run the effluent directly to the toilets. The drainage line should be clearly marked on the floor and thoroughly secured, to prevent accidental rupture and leaks.

Disinfection of dialysis effluent is not routine in the care of patients with viral or bacterial infections in general, and is not recommended by the CDC for EVD patients unless mandated by local wastewater departments or public health care departments. If disinfection is required, then quaternary ammonium, bleach, or other disinfectant may be added to the effluent bags or to the toilet prior to flushing.

\section{Additional Resources}

Additional resources for RRT planning in EVD include: (1) CDC recommendations for safely performing acute hemodialysis in patients with Ebola virus disease in US hospitals. Available online at http://www.cdc.gov/ vhf/ebola/hcp/guidance-dialysis.html., and (2) from The American Society of Nephrology: http://www.asn-online. org/news/2014/ASN_FAQs_Ebola_Virus_Disease_and_ Dialysis.pdf.

\section{Screening for Ebola in the General and the Outpatient} Dialysis Unit

At this point in the African epidemic, travel to an area where outbreaks are ongoing is the first epidemiologic link needed to identify individuals at risk for possible infection. The CDC has instituted procedures at five major airports in the United States to screen travelers that have been identified as travelling to the African countries with ongoing Ebola virus disease outbreaks. Individuals are classified as high risk, some risk, low (but not zero risk), and no identifiable risk. High risk includes individuals who have had exposure to blood or bodily fluids of a per- son with symptomatic EVD without the use of appropriate PPE or standard biosafety precautions, individuals with direct care of a symptomatic person in the household or direct contact with a dead body without appropriate PPE. Most returning health care workers will fall under the categorization of 'some risk'. This includes individuals with direct contact with symptomatic Ebola patients or their bodily fluids while using appropriate PPE or prolonged close contact with a symptomatic patient without PPE. 'Low (but not zero) risk' includes individuals without known exposures but who have traveled within the past 21 days to countries with widespread transmission of Ebola or brief proximity or contact with a symptomatic patient early in the disease course ('Epidemiologic Risk Factors to Consider when Evaluating a Person for Exposure to Ebola Virus', Centers for Disease Control and Prevention, 2014. http://www.cdc.gov/vhf/ ebola/exposure/risk-factors-when-evaluating-personfor-exposure.html). Based on this risk stratification, state and local health departments are notified of the individuals' risk and individuals are monitored for fever or development of symptoms.

Screening for Ebola virus by hospitals in general is a necessity, but is even more important for chronic dialysis facilities where Ebola virus has the potential to spread rapidly and easily in a dialysis unit. It has been recommended that all health care facilities use the triage algorithm, 'Identify, Isolate and Inform' for all individuals being seen in their facilities regardless of the reason for the visit - which can be applied even to the outpatient dialysis unit. This entails identifying individuals with exposure history in the past 21 days and identifying if they are having clinical symptoms of Ebola. If they have both exposure history and symptoms, the individual should be isolated and PPE should be employed and the infection prevention department and health department should be notified immediately. For chronic dialysis patients, this will likely require inpatient admission and receipt of dialysis in an isolation unit ('Identify, Isolate, Inform: Emergency Department Evaluation and Management for Patients Who Present with Possible Ebola Virus Disease', Centers for Disease Control and Prevention, 2014. http:// www.cdc.gov/vhf/ebola/hcp/ed-management-patientspossible-ebola.html).

In conclusion, with appropriate planning and protocols, provision of advanced supportive care including renal replacement therapy may be provided safely and to patient benefit. Provision for RRT should be considered in hospitals that are planning to accept the care of patients with EVD. 


\section{References}

1 Dixon MG, Schafer IJ: Ebola viral disease outbreak - West Africa, 2014. MMWR Morb Mortal Wkly Rep 2014;63:548-551.

2 World Health Organization: Geographic distribution of Ebola virus disease outbreaks in humans and animals - 2014. http://www. who.int/csr/disease/ebola/global_ebolaout breakrisk_20140818-1.png?ua=1 (accessed December 2, 2014).

3 World Health Organization: Ebola virus disease, Fact sheet Number 103. http://www. who.int/mediacentre/factsheets/fs103/en/. (accessed December 2, 2014).

4 World Health Organization: Ebola response roadmap situation report. http://www.who. int/csr/disease/ebola/situation-reports/en/. (accessed December 17, 2014).

-5 Fowler RA, Fletcher T, Fischer WA 2nd, et al: Caring for critically ill patients with Ebola virus disease. Perspectives from West Africa. Am J Respir Crit Care Med 2014;190:733737.

6 Lyon GM, Mehta AK, Varkey JB, et al: Clinical care of two patients with Ebola virus disease in the United States. N Engl J Med 2014; 37:2402-2409.

7 Fletcher TE, Fowler RA, Beeching NJ: Understanding organ dysfunction in Ebola virus disease. Intensive Care Med 2014;40:19361939.

8 Rollin PE, Bausch DG, Sanchez A: Blood chemistry measurements and D-Dimer levels associated with fatal and nonfatal outcomes in humans infected with Sudan Ebola virus. J Infect Dis 2007;196(suppl 2):S364S371.

-9 Connor MJ Jr, Kraft C, Mehta AK, et al: Successful delivery of RRT in Ebola virus disease. J Am Soc Nephrol 2015;26:31-37.

10 Martines RB, Ng DL, Greer PW, Rollin PE, Zaki SR: Tissue and cellular tropism, pathology and pathogenesis of Ebola and Marburg viruses. J Pathol 2014;253:153-174.
11 Kreuels B, Wichmann D, Emmerich P, et al: A case of severe Ebola virus infection complicated by gram-negative septicemia. N Engl J Med 2014;371:2394-2401.

12 Bausch DG, Towner JS, Dowell SF, et al: Assessment of the risk of Ebola virus transmission from bodily fluids and fomites. J Infect Dis 2007;196(suppl 2):S142-S147.

13 Feldmann H, Geisbert TW: Ebola haemorrhagic fever. Lancet 2011;377:849-862.

14 Rowe AK, Bertolli J, Khan AS, et al; Commission de Lutte contre les Epidemies a Kikwit: Clinical, virologic, and immunologic followup of convalescent Ebola hemorrhagic fever patients and their household contacts, Kikwit, Democratic Republic of the Congo. J Infect Dis 1999;179(suppl 1):S28-S35.

15 Towner JS, Rollin PE, Bausch DG, et al: Rapid diagnosis of Ebola hemorrhagic fever by reverse transcription-PCR in an outbreak setting and assessment of patient viral load as a predictor of outcome. J Virol 2004;78:43304341.

16 Simon V, Ho DD, Abdool Karim Q: HIV/ AIDS epidemiology, pathogenesis, prevention, and treatment. Lancet 2006;368:489504.

17 Smith PW, Anderson AO, Christopher GW, et al: Designing a biocontainment unit to care for patients with serious communicable diseases: a consensus statement. Biosecur Bioterror 2006;4:351-365.

18 Hill CE, Burd EM, Kraft CS, et al: Laboratory test support for ebola patients within a highcontainment facility. Lab Med 2014;45:e109e111.

19 Centers for Disease Control and Prevention: Interim guidance for environmental infection control in hospitals for Ebola virus. http:// www.cdc.gov/vhf/ebola/hcp/environmentalinfection-control-in-hospitals.html. (accessed December 2, 2014).
20 Centers for Disease Control and Prevention: Recommendations for safely performing Acute Hemodialysis in Patients with Ebola Virus Disease in U.S. Hospitals. http://www. cdc.gov/vhf/ebola/hcp/guidance-dialysis. html. (accessed October 23, 2014).

21 Callegari J, Antwi S, Wystrychowski G, Zukowska-Szczechowska E, Levin NW, Carter M: Peritoneal dialysis as a mode of treatment for acute kidney injury in sub-Saharan Africa. Blood Purif 2013;36:226-230.

22 Kidney Disease: Improving Global Outcomes (KDIGO) Acute Kidney Injury Work Group. KDIGO Clinical Practice Guideline for Acute Kidney Injury. Kidney Int Suppl 2012;2:1-138.

23 van de Wetering J, Westendorp RG, van der Hoeven JG, Stolk B, Feuth JD, Chang PC: Heparin use in continuous renal replacement procedures: the struggle between filter coagulation and patient hemorrhage. J Am Soc Nephrol 1996;7:145-150.

24 Tolwani AJ, Wille KM: Anticoagulation for continuous renal replacement therapy. Semin Dial 2009;22:141-145.

25 Biancofiore G, Esposito M, Bindi L, et al: Regional filter heparinization for continuous veno-venous hemofiltration in liver transplant recipients. Minerva Anestesiol 2003;69: 527-534; discussion 534-538.

26 Clark WR, Turk JE, Kraus MA, Gao D: Dose determinants in continuous renal replacement therapy. Artif Organs 2003;27:815-820.

27 Davies HT, Leslie G, Pereira SM, Webb SA: A randomized comparative crossover study to assess the affect on circuit life of varying predilution volume associated with $\mathrm{CVVH}$ and CVVHDF. Int J Artif Organs 2008;31:221227.

28 Hofmann CL, Fissell WH: Middle-molecule clearance at 20 and $35 \mathrm{ml} / \mathrm{kg} / \mathrm{h}$ in continuous venovenous hemodiafiltration. Blood Purif 2010;29:259-263. 